\title{
Lung Dendritic Cell Developmental Programming, Environmental Stimuli, and Asthma in Early Periods of Life
}

\author{
Shanjana Awasthi, ${ }^{1}$ Bhupinder Singh, ${ }^{1}$ Robert C. Welliver, ${ }^{2}$ and Rodney R. Dietert ${ }^{3}$ \\ ${ }^{1}$ Department of Pharmaceutical Sciences, College of Pharmacy, University of Oklahoma Health Sciences Center, \\ Oklahoma City, OK 73117, USA \\ ${ }^{2}$ Department of Pediatrics, University of Oklahoma Health Sciences Center, Oklahoma City, OK 73104, USA \\ ${ }^{3}$ Department of Microbiology and Immunology, Cornell University, Ithaca, NY 14853, USA
}

Correspondence should be addressed to Shanjana Awasthi, shanjana-awasthi@ouhsc.edu

Received 1 June 2012; Revised 29 September 2012; Accepted 30 September 2012

Academic Editor: Hamida Hammad

Copyright ( $) 2012$ Shanjana Awasthi et al. This is an open access article distributed under the Creative Commons Attribution License, which permits unrestricted use, distribution, and reproduction in any medium, provided the original work is properly cited.

Dendritic cells (DCs) are important cells of our innate immune system. Their role is critical in inducing adaptive immunity, tolerance, or allergic response in peripheral organs_lung and skin. The lung DCs are not developed prenatally before birth. The DCs develop after birth presumably during the first year of life; exposures to any foreign antigen or infectious organisms during this period can significantly affect DC developmental programming and generation of distinct DC phenotypes and functions. These changes can have both short-term and long-term health effects which may be very relevant in childhood asthma and predisposition for a persistent response in adulthood. An understanding of DC development at molecular and cellular levels can help in protecting neonates and infants against problematic environmental exposures and developmental immunotoxicity. This knowledge can eventually help in designing novel pharmacological modulators to skew the DC characteristics and immune responses to benefit the host across a lifetime.

\section{Introduction}

Asthma is a serious pulmonary disease that affects about 300 million people worldwide [1], and 8.2\% (about 25 million) of the population within the USA [2]. A significant number of patients develop asthma during early childhood. A number of cross-sectional and longitudinal cohort studies in adult asthmatic patients suggest that the childhood asthma poses a risk for more severe asthma or relapse during adulthood [3-6]. One among ten children has asthma, and this trend has increased over the recent years [7]. Characteristics (airway obstruction, airway hyperresponsiveness, atopy, and recent wheeze) observed in children have been reported as predictors of asthma symptoms in adulthood. This is supported by evidence that the sensitization to allergens at young age increases the likelihood of asthma in adulthood [8-11].

In allergic asthma, an immune reaction is caused by inhaled allergens with an overwhelming inflammatory response and obstruction in the airways. As a first step in sensitization, the antigen presenting cells (dendritic cellsDCs, macrophages, and lung epithelial cells) take up and process the inhalant allergens. The DCs are recognized as the key immune sentinel cell in the peripheral organs, including lung [12], and are at the cross-roads of inducing tolerance or inflammation [13]. The DCs are activated directly or via cell-cell interaction [14]. The activated DCs in turn stimulate $\mathrm{T}$ and $\mathrm{B}$ cells and other immune cells, which release a variety of cytokines, chemokines, and chemical mediators. These mediators are responsible for affecting the local microenvironment and generating inflammation and obstruction in airways. Traditionally, asthma has been known as the Th2-mediated disease (Figure 1). Both Th2and non-Th2-dependent immune elements and mechanisms are now recognized for a number of phenotypes and endotypes of asthma [15-17].

While asthma phenotypes and endotypes are not fully characterized, resident lung DC types could be important $[12,18-22]$. Lung DCs exhibit unique phenotypes than 


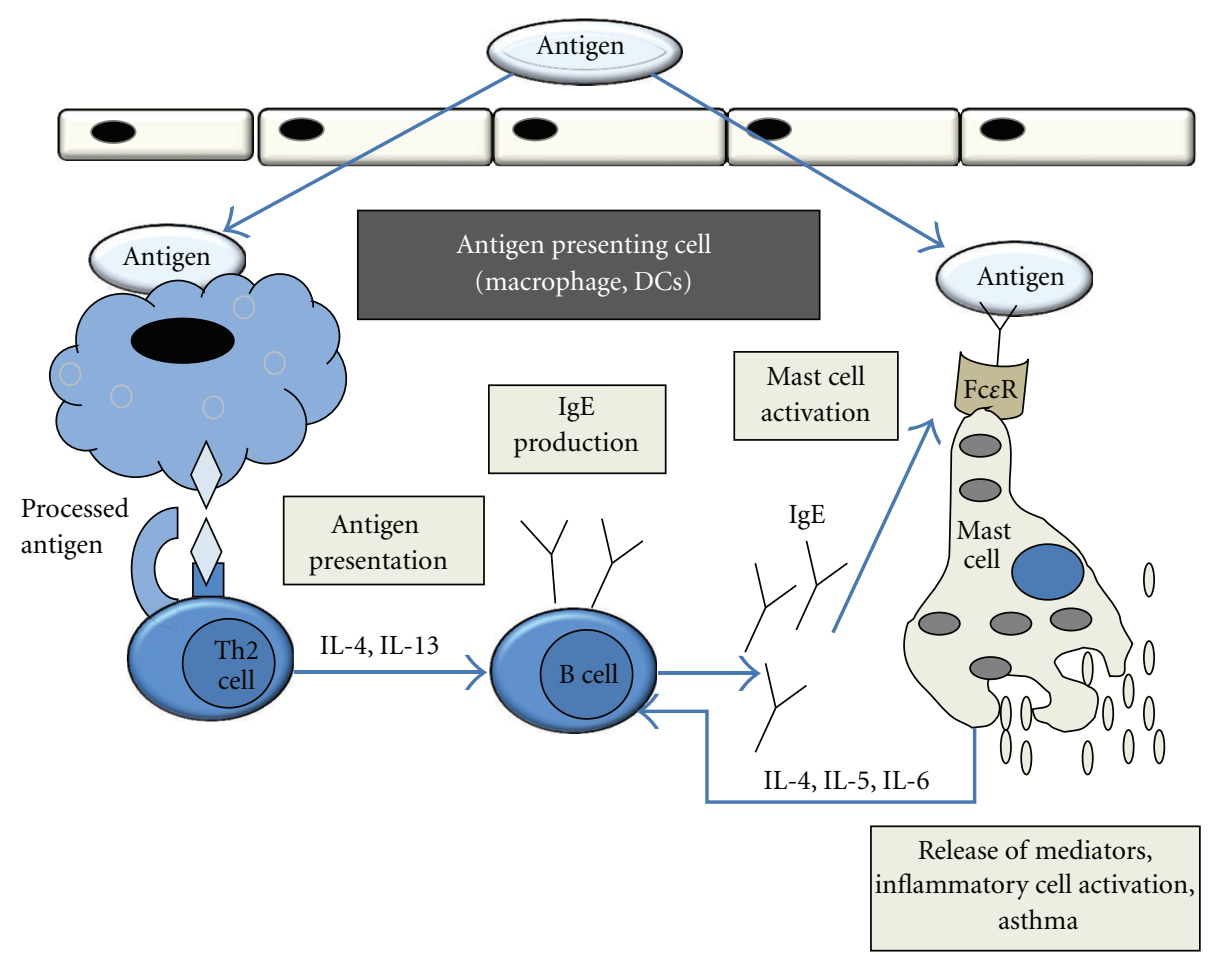

FIgURE 1: An illustration depicting the types of immune cells involved in a Th2-mediated allergic response. Antigen presenting cells (macrophages or DCs) take up the antigen, process it, and present it on the MHC molecule on the cell surface. The antigen presenting cells induce naïve T cells towards Th1 or Th2. Th2 response is mainly responsible for downstream events that include activation of B cells, production of IgE, and binding of IgE to FcE receptor on the cell surface of mast cells, resulting into mast cell degranulation and inflammation.

those present in other organs or in circulation [23], and are distributed throughout the alveolar epithelium, alveolar parenchyma, and nasal mucosa $[24,25]$. A variety of investigations in rodents and human patients have reported the importance of different lung DC types in asthma [26-29]. This corresponds with the reported results on alterations in selective DC populations in the bronchoalveolar lavage fluids (BALFs) and in peripheral blood of patients with asthma (Table 1). A number of distinctive reports are available in the literature on the characteristics and functions of lung DC types $[23,30,31]$, and are not reviewed here. In this paper, we provide an overview of lung DC development, exposure to pathogens, allergens, and environmental chemicals during early childhood, and their long-term impact on asthma development.

\section{Critical Window of Immune Vulnerability}

Over the last several years, prominent research studies have demonstrated that the late fetal and early postnatal periods are phases of reduced immune competence $[12,32,33]$. This reported reduced immune competence corresponds with the immaturity of immune system. In particular, the lung DCs are underdeveloped prenatally and close to term at birth [18, 34-38]. The development of lung DCs during infanthood has not been studied. Concurrent exposures to allergens and other environmental factors during this "critical window of immune vulnerability" have the potential to program DC types, DC functions, and DC-mediated tolerance or sensitization, which can have long-term respiratory and immunological consequences (Figure 2) [32, 39, 40].

\section{Developing Immune System and Exposure to Asthma-Triggering Agents in Early Childhood}

Particular events during early childhood can set the stage for specific developmental programming of DCs. The flexibility of a developing immune system and simultaneous exposure to allergens and other environmental stimuli can be important compounding factors for both the establishment and a long-term persistence of asthma.

In addition to allergens, other risk factors for DC developmental programming towards particular DC phenotype and function during this "critical window period" may include environmental chemicals, drugs, certain dietary factors, infectious agents, and physical and psychological stressors. Not surprisingly, research studies suggest that the maturation and function of DCs are shifted by some of these predisposing risk factors. These include heavy metals, such as lead [41], and air pollutants particularly those from traffic [42] and environmental tobacco smoke [43]. Among relevant shifts that have been reported are: (1) reduced expression of Toll-like receptors (TLR)2 and TLR4 [44], (2) shift to 
TABLE 1: DC subsets in patients with asthma.

\begin{tabular}{|c|c|c|}
\hline Clinical Condition & Altered DC phenotypes & References \\
\hline Asthma & $\begin{array}{l}\text { pDC (HLA-DR+, CD123+) increased in BALF } \\
\text { mDC (HLA-DR+, CD11c+) increased in BALF }\end{array}$ & [99] \\
\hline Allergic asthma & $\mathrm{mDC}(\mathrm{BDCA}-3+$, mannose receptor + ) increased in BALF & {$[100]$} \\
\hline Allergic asthma & $\begin{array}{l}\text { pDC (BDCA4+) with increased FceRI in blood } \\
\text { mDC }(\mathrm{CD} 1 \mathrm{c}+) \text { with increased FceRI in blood }\end{array}$ & {$[101,102]$} \\
\hline Asthma & Increased DC proportions in peripheral blood & {$[103]$} \\
\hline Allergic asthma patients challenged with allergen & Increased $\mathrm{pDC}$ and $\mathrm{mDC}$ in sputum & {$[104]$} \\
\hline Asthma & Increase in $\mathrm{pDC} 1$ and $\mathrm{pDC} 2$ expressing FceRI & {$[105]$} \\
\hline Repeated exposure to allergen & Depletion of mDCs & {$[23]$} \\
\hline Asthmatic children & DC2 (CD11c-, CD123high+) decreased in blood & {$[106]$} \\
\hline Asthmatic patients & Increased CD1a+ cells in bronchial mucosa & {$[107]$} \\
\hline Experimentally elicited allergic rhinitis & pDC increased in nasal mucosa & {$[108]$} \\
\hline Asthma & pDC increased; decreased $\mathrm{mDC}: \mathrm{pDC}$ ratio in blood & {$[109]$} \\
\hline Children with asthma & Deficiency of circulating pDC & {$[110]$} \\
\hline Atopic patients with chronic rhinosinusitis & Increased FceRI on DC (CD1+) & {$[111]$} \\
\hline
\end{tabular}

Abbreviations: BALF: bronchoalveolar lavage fluid, mDC: myeloid DC, pDC: plasmacytoid DC, BDCA: blood dendritic cell antigen.

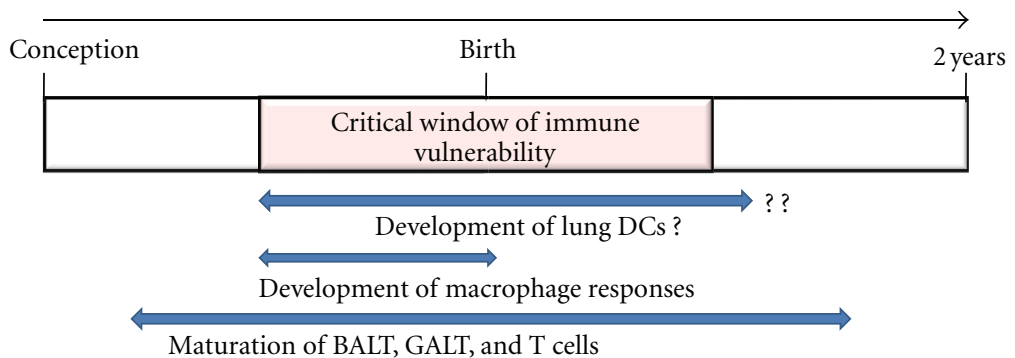

(a)

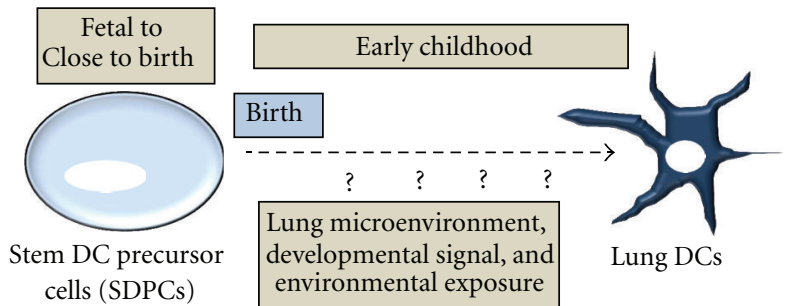

(b)

FIGURE 2: Immune development during critical window of vulnerability. (a) Timeline of maturation of bronchus-associated lymphoid tissue (BALT), gut-associated lymphoid tissue (GALT), T cells, and macrophages. (b) Lung-resident SDPCs could be the plausible source of lung DCs in early childhood. The timing of lung DC development, environmental factors triggering this transition, and signaling mechanisms involved in DC development remain unknown.

Th2-biased adaptive immune response [45], and (3) promotion of misregulated (unresolved) inflammation $[46,47]$.

A number of recent studies have demonstrated that early exposures to Chlamydia muridarum (an intracellular pathogen) [48-50], Bacillus Calmette-Guérin (BCG) [51], and influenza $A$ virus [52] alter the immune responses against allergens in adulthood via affecting the DC types and functions. Bacterial infections or stimulation with TLR4 ligand (Gram-negative bacteria-derived lipopolysaccharide) have been shown to skew the T-cell response to Th1 type during childhood [53]. In this regard, "Probiotics" and "Hygiene hypothesis" have been discussed elsewhere in the literature for controlling asthma-related immune response [54, 55].

\section{Respiratory Syncytial Virus (RSV) Infection and Asthma}

Respiratory syncytial virus infection is the most common cause of bronchiolitis and pneumonia in children under 1 year of age (Centers for Disease Control and Prevention, Atlanta, GA, USA). Severe forms of the RSV lower respiratory 
tract infections (LRTI) are characterized by airway obstruction and prominent wheezing. Furthermore, RSV infection in infancy has been linked to the development of asthma in childhood. Thus, there has been great interest in determining whether the pathogenesis of RSV bronchiolitis in infancy induces a persistent Th2 bias, leading to the development of Th2-dependent asthma in later childhood.

Some studies have focused on the expression of the prototype Th1 cytokine, interferon gamma (IFN- $\gamma$ ), and the Th2 cytokine, interleukin-4 (IL-4). Bendelja and colleagues studied the expression of IFN- $\gamma$ and IL-4 in peripheral blood lymphocytes (PBL) of infants with various forms of RSV infection [56]. Among RSV-infected infants, the percentage of PBL positive for IL-4 was slightly greater than the percentage positive for IFN- $\gamma$, thus suggesting a Th2 bias. However, the expression of IL- 4 was greater in subjects with mild upper respiratory tract infection (URTI) than in subjects with bronchiolitis or pneumonia. IFN- $\gamma$ expression remained unaffected. Therefore, patterns of IFN- $\gamma$ and IL-4 expression by PBL could not be associated with the severity of RSV infection.

Others have determined the quantities of IFN- $\gamma$ and IL-4 cytokines in respiratory tract secretions of infants with RSV infections. In one study, IFN- $\gamma$ was found to be the predominant cytokine in subjects with all forms of respiratory tract illness related to RSV infection, with slightly higher ratios of IFN- $\gamma$ to IL-4 in those with LRTI in comparison to those with URTI alone [57]. A second, larger study similarly demonstrated that the Th2 cytokines, IL-4, IL-5, and IL-13, were usually undetectable in secretions from infants with all forms of RSV infection. IFN- $\gamma$ appeared to be protective against severe illness, in that IFN- $\gamma$ concentrations were greater in subjects with milder, nonhypoxic forms of RSV-induced LRTI than in those with more severe LRTI accompanied by hypoxia [58]. In all of these studies, the differences in ratios of IFN- $\gamma$ to Th2 cytokines were determined only by variations in IFN- $\gamma$ concentrations between the groups. The findings of subsequent studies have also suggested a protective role for IFN $-\gamma$ in RSV infection of infants $[59,60]$.

How the DC phenotypes and DC-induced T-cell responses are skewed following RSV infection is an important question, and could provide clues to the severity of the disease and predisposition to asthma. Infection of human infants with RSV and other viruses is followed by the appearance of DCs in nasopharyngeal and tracheal secretions $[61,62]$. RSV infection of monocyte-derived DCs causes maturation of the cells, with expression of costimulatory molecules that participate in the instruction of $\mathrm{T}$ cells. However, it results in impaired CD4-positive T cells [63]. Although DC types were not addressed, the lung tissues from infants with fatal RSV demonstrated a lack of CD8-positive $\mathrm{T}$ cells [64]. Studies in mice have suggested unique roles for myeloid (mDC) and plasmacytoid DCs (pDC) in RSV infection. The pDCs in RSV-infected mice reduce the viral replication, while depletion of $\mathrm{pDCs}$ results in enhanced inflammatory responses and greater airway hyperreactivity [65]. A balance between $\mathrm{mDC}$ and $\mathrm{pDC}$ seems to determine the immune responses to RSV and airway reactivity following RSV infection [66]. The recruitment and activity of DC subsets occurring after RSV infection could skew immune responses toward either Th1 or Th2 cytokine pathways, thereby determining the eventual development of atopic disease or long-term airway hyperreactivity following RSV infection in infancy.

\section{Lung DC Development}

Despite growing understanding about the DC characteristics and functions in adult patients and animal models, the natural processes of lung DC development in prenatal or neonatal phase, as well as differentiation, maturation, and functional specialization of DCs, have not yet been studied. An understanding of perinatal and infant DC maturation in environmentally exposed tissues (including lung) is critical to better manage immune maturation for a healthier life course.

Histological details reveal that the MHC class II-positive cells start to appear in lung tissues of rat and human fetuses at $30-58 \%$ of term. Since the MHC class II is expressed ubiquitously by a variety of immune and nonimmune cells, the interpretation may not be DC specific. The appearance of MHC class II-positive DCs increases only after birth [37, 67-70].

Importantly, the airway structures and epithelial cell system are also not fully developed at the time of birth or during the neonatal period. It is reasonable to believe that direct cellcell interaction or chemical mediators and growth factors released by another cell type can have a significant impact on the establishment of normal lung DC infrastructure as the lung microenvironment and the normal lung physiology evolve $[13,71,72]$. Repeated exposure to potentially harmful allergens or other environmental stimuli during this period can significantly affect the DC programming, phenotypes, and functions. Although it remains to be studied, these events may prompt a long-term memory for the generation of asthma-promoting DCs.

\section{Animal Models for Studying Lung DC Development}

Technical and ethical issues related to the availability of neonatal and infant lung tissues limit the enthusiasm to conduct studies that address the issues related to lung DC development. Studies are limited to first challenging the neonatal animal with infectious or allergenic stimuli and then investigating the DC types and functions later in life in the same animal. This approach may not adequately reflect the dynamic process of DC development or programming during early childhood in humans. Since DCs make $<1 \%$ of total lung cells [34], it is not possible to harvest sufficient DC populations from rodent pups because of their smallsized lung. Hundreds of small-sized, age-matched mouse pups (most commonly used model) would be needed to harvest an adequate number of cells; it is almost impossible to have simultaneous births and enough age-matched mouse progeny available for this purpose. Also, the cells cannot be 
pooled from pups born close together, because murine pups (between birth and one-month) age at 150 times faster rate than humans [73]. There are significant differences in the lung anatomical [74], developmental, and immunological aspects of humans and mice (e.g., the lymphocyte and neutrophil distribution in blood, DC phenotypes) which make it difficult to interpret and translate the results to human infants [75-77]. As such, murine DC precursors/DC phenotypes are different from those reported in humans $[23,78]$. Cellular intermediates within the hematopoietic stem cell hierarchy tree have been identified in tissues of mice and humans $[79,80]$; significant differences have been noted in regards to their subsets and frequency [78]. Lack of reagents and paucity of information on DC-precursors or DCs limit such studies in other rodents. These studies are also not possible in human neonates or infants due to ethical reasons. Large animal models are expensive and require diligent work; these models can mimic the conditions of human infants very closely. To this effect, an asthma model is available in Rhesus monkeys. Similarities have been reported in asthmatic response among Rhesus monkeys and humans; the house dust mite antigen induces asthma conditions with clinical profiles; the biochemical and immunological markers are similar to those in human patients of asthma $[74,81,82]$.

The baboon model seems ideal for studying the early human immune maturation and the developmental programming [83] because of similarities in ontogeny, immunology, reproductive physiology, placentation, and maternalfetal transfer [84-88]. They are very close to humans in the evolutionary tree [89], and the lung development pattern in preterm baboons is similar to that found in preterm human babies [90]. Some of the immunological aspects [91-93], the bronchoconstriction, and the airway response against platelet-activating factor [94] in baboons are also analogous to those of humans and asthmatic patients, respectively. The advantages of the baboon over other commonly used primates, such as Rhesus monkey, include the ease of timed pregnancies due to the estrogen-sensitive sex skin in cycling females, the availability (baboons breed year-round), and the relative ease of handling (reviewed in [91]). Moreover, it allows harvesting of sufficient number of cells of interest from relatively large tissues of neonate and infant baboons.

We have studied the development of pulmonary innate immunity, including DCs, in a non human primate baboon (Papio species) model [34, 35, 95-97]. We have investigated the DC phenotypes and functions in prematurely delivered and close-to-term baboons (67-95\% of gestation). Our results demonstrate that lung DC population having low density (similar to those of adult baboon lung DC population) remains underdeveloped until close to birth [34]. Since we do not know the stage of differentiation and the cell subsets, it is probably more appropriate to call them stem DC precursor cells (SDPCs) [36]. We have recently observed that the SDPCs can differentiate into DCs in vitro under DC-promoting conditions (Figure 3). A significant increase in expression of DC markers and tentacles is observed over time. It has been proposed by others that the tissue DCs can be generated from hitherto unknown resident stem cell populations. Isolation of stem cells has been reported from adult human lung [98]; their differentiation into lung DCprecursors or DCs has not yet been studied.

\section{Molecular and Immunologic Basis for DC Programming and Therapeutic Opportunities}

In summary, our results indicate that the lung DCs are not developed at least until birth. We do not know when the DCs develop after birth during childhood, what triggers the lung DC development, and how the pathogenic stimuli affect the DC development leading to DC phenotypes with different functions. We speculate that pathogenic stimuli, such as allergens, may alter the lung DC developmental programming or SDPC $\rightarrow$ DC transition during early childhood in a way that an immunological memory is created for generation of asthma-promoting DC phenotypes or immune responses. Persistent reexposure to allergens may bolster the generation of these DC phenotypes so that an allergic response is maintained for a long time.

An understanding of the basic immunobiology of DC development and the early programming of asthmapromoting DC phenotypes and functions against allergenic stimuli can pave the way to identify the basis of childhood asthma. Although it can be challenging to study the molecular mechanisms for lung DC development in vivo, studies with baboon lung SDPCs and DCs can provide useful tools to unravel the molecular and immunologic basis of the lung DC development and develop novel pharmacological modulators. A focused effort in this direction can provide a unique opportunity to effectively manage the pediatric immune system for optimized maturation and reduced health risks. This would include opportunities to intervene at an early age and skew the DC programming towards normal DC phenotype and function using pharmacological modulators. Additionally, this information may be useful in better protecting neonates and infants from environmental insults that increase the later-life health risks. It is the magnitude and persistence of downstream immunoinflammatory effects of tissue DC function that position this topic as a central health issue for allergic and other chronic diseases.

\section{Funding}

Funding was provided by the American Lung Association and Presbyterian Health Foundation to S. Awasthi for conducting related studies in baboons.

\section{Authors' Contribution}

All authors have contributed and reviewed the paper. Specific contributions by the authors are as follows: B. Singh compiled Table 1 in the paper. R. C. Welliver and R. R. Dietert contributed to the text related to RSV, critical window of immune vulnerability, and environmental exposures, respectively. S. Awasthi conducted studies related to the baboon DCs in her lab and coordinated with co-authors for compilation of this paper. 

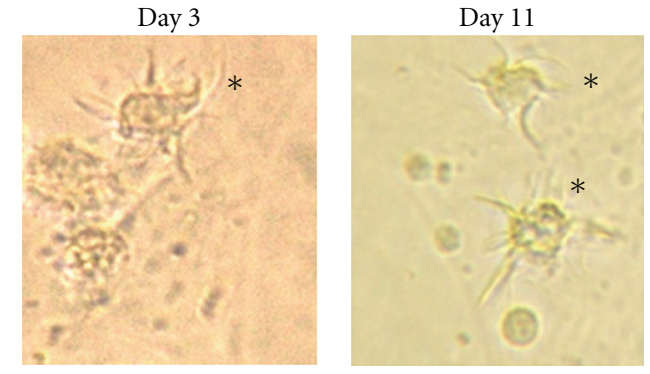

(a)
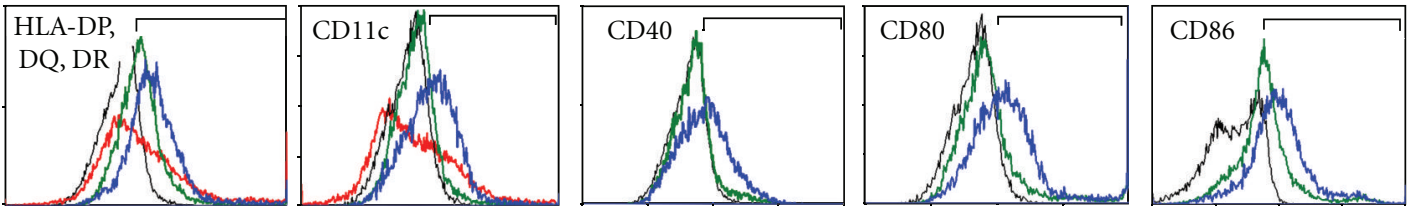

(b)

\begin{tabular}{|l|l|l|l|l|l|}
\hline $\begin{array}{l}\text { Markers } \\
\text { Days in culture }\end{array}$ & $\begin{array}{c}\text { HLA- } \\
\text { DP, DQ, DR }\end{array}$ & CD11c & CD40 & CD80 & CD86 \\
\hline Isotype control & $4.4 \%(160)$ & $9 \%(148)$ & $4.4 \%(160)$ & $9 \%(148)$ & $5.3 \%(79)$ \\
\hline 6 days & $30 \%(366)$ & $19 \%(355)$ & $10 \%(280)$ & $28 \%(1601)$ & $39 \%(328)$ \\
\hline 11 days & $37 \%(629)$ & $44 \%(322)$ & $26 \%(382)$ & $54 \%(1049)$ & $61 \%(295)$ \\
\hline 17 days & $41 \%(617)$ & $34 \%(355)$ & Not done & Not done & Not done \\
\hline
\end{tabular}

(c)

FIGURE 3: The SDPCs harvested from a close-to-term fetal baboon differentiate into DCs when cultured in presence of GM-CSF, IL-4, and TNF- $\alpha$. The lung SDPCs were harvested on OptiPrep density gradient as per the method published earlier [34]. (a) Photomicrograph showing cells with dendrites $\left(^{*}\right)$. (b) Flow cytometry data showing increase in DC-marker expression. Black line: isotype control antibodystained cells, green line: 6 days, blue line: 11 days, red line: 17 days — cells stained with antibodies to particular marker. (c) Data in the table shows \% cells (fluorescent intensity) gated in the marked region of histogram charts in (b) staining positive for the specific marker.

\section{Acknowledgments}

The authors acknowledge the Baboon Research Resources at the University of Oklahoma Health Sciences Center, Oklahoma City, OK, USA, and Baboon resource programs at the University of Texas Health Sciences Center and Southwest Foundation for Biomedical Research, San Antonio, TX, USA, for providing baboon tissues and fluid specimens.

\section{References}

[1] World Health Organization, Global Surveillance, Prevention and Control of Chronic Respiratory Diseases: A Comprehensive Approach, 2007.

[2] National Center for Health Statistics, 2012, http://www.cdc .gov/nchs/data/nhsr/nhsr032.pdf.

[3] C. Ségala, G. Priol, D. Soussan et al., "Asthma in adults: comparison of adult-onset asthma with childhood-onset asthma relapsing in adulthood," Allergy, vol. 55, no. 7, pp. 634-640, 2000.

[4] M. R. Sears, J. M. Greene, A. R. Willan et al., "A longitudinal, population-based, cohort study of childhood asthma followed to adulthood," The New England Journal of Medicine, vol. 349, no. 15, pp. 1414-1422, 2003.
[5] L. Duijts, "Fetal and infant origins of asthma," European Journal of Epidemiology, vol. 27, no. 1, pp. 5-14, 2012.

[6] B. Brunekreef, E. Von mutius, G. K. Wong, J. A. Odhiambo, and T. O. Clayton, "Early life exposure to farm animals and symptoms of asthma, rhinoconjunctivitis and eczema: an ISAAC phase three study," International Journal of Epidemiology, vol. 41, no. 3, pp. 753-761, 2012.

[7] L. Akinbami, "The state of childhood asthma, United States, 1980-2005," Advance data, no. 381, pp. 1-24, 2006.

[8] B. G. Toelle, W. Xuan, J. K. Peat, and G. B. Marks, "Childhood factors that predict asthma in young adulthood," European Respiratory Journal, vol. 23, no. 1, pp. 66-70, 2004.

[9] C. Almqvist, Q. Li, W. J. Britton et al., "Early predictors for developing allergic disease and asthma: examining separate steps in the 'allergic march"' Clinical and Experimental Allergy, vol. 37, no. 9, pp. 1296-1302, 2007.

[10] T. Schäfer, G. Wölke, J. Ring, H. E. Wichmann, and J. Heinrich, "Allergic sensitization to cat in childhood as major predictor of incident respiratory allergy in young adults," Allergy, vol. 62, no. 11, pp. 1282-1287, 2007.

[11] L. M. Taussig, A. L. Wright, C. J. Holberg, M. Halonen, W. J. Morgan, and F. D. Martinez, “Tucson Children's respiratory study: 1980 to present," Journal of Allergy and Clinical Immunology, vol. 111, no. 4, pp. 661-675, 2003. 
[12] P. G. Holt and P. D. Sly, "Prevention of allergic respiratory disease in infants: current aspects and future perspectives," Current Opinion in Allergy and Clinical Immunology, vol. 7, no. 6, pp. 547-555, 2007.

[13] B. N. Lambrecht and H. Hammad, "The role of dendritic and epithelial cells as master regulators of allergic airway inflammation," The Lancet, vol. 376, no. 9743, pp. 835-843, 2010.

[14] B. N. Lambrecht and H. Hammad, "The other cells in asthma: dendritic cell and epithelial cell crosstalk," Current Opinion in Pulmonary Medicine, vol. 9, no. 1, pp. 34-41, 2003.

[15] S. Wenzel, "Severe asthma: from characteristics to phenotypes to endotypes," Clinical and Experimental Allergy, vol. 42, no. 5, pp. 650-658, 2012.

[16] N. R. Bhakta and P. G. Woodruff, "Human asthma phenotypes: from the clinic, to cytokines, and back again," Immunological Reviews, vol. 242, no. 1, pp. 220-232, 2011.

[17] S. E. Wenzel, "Asthma phenotypes: the evolution from clinical to molecular approaches," Nature Medicine, vol. 18, no. 5, pp. 716-725, 2012.

[18] P. G. Holt, "Dendritic cell ontogeny as an aetiological factor in respiratory tract diseases in early life," Thorax, vol. 56, no. 6, pp. 419-420, 2001.

[19] P. G. Holt, C. Macaubas, S. L. Prescott, and P. D. Sly, "Microbial stimulation as an aetiologic factor in atopic disease," Allergy, vol. 54, supplement 49, pp. 12-16, 1999.

[20] P. G. Holt, J. Oliver, N. Bilyk et al., "Downregulation of the antigen presenting cell function(s) of pulmonary dendritic cells in vivo by resident alveolar macrophages," The Journal of Experimental Medicine, vol. 177, no. 2, pp. 397-407, 1993.

[21] P. G. Holt and J. W. Upham, "The role of dendritic cells in asthma," Current Opinion in Allergy and Clinical Immunology, vol. 4, no. 1, pp. 39-44, 2004.

[22] A. Rate, J. W. Upham, A. Bosco, K. L. McKenna, and P. G. Holt, "Airway epithelial cells regulate the functional phenotype of locally differentiating dendritic cells: implications for the pathogenesis of infectious and allergic airway disease," Journal of Immunology, vol. 182, no. 1, pp. 72-83, 2009.

[23] M. H. Grayson, "Lung dendritic cells and the inflammatory response," Annals of Allergy, Asthma and Immunology, vol. 96, no. 5, pp. 643-651, 2006.

[24] B. J. Masten and M. F. Lipscomb, "Methods to isolate and study lung dendritic cells," in Lung Macrophages and Dendritic Cells in Health and Disease, M. F. Lipscomb and S. W. Russell, Eds., pp. 223-238, Marcel Dekker, New York, NY, USA, 1997.

[25] A. S. McWilliam and P. G. Holt, "Immunobiology of dendritic cells in the respiratory tract: steady-state and inflammatory sentinels?" Toxicology Letters, vol. 102-103, pp. 323329, 1998.

[26] B. N. Lambrecht, "Lung dendritic cells: from basic physiologyto clinical applications," Acta Clinica Belgica, vol. 62, no. 5, pp. 330-334, 2007.

[27] B. Lambrecht and H. Hammad, "Lung dendritic cells: targets for therapy in allergic disease," Chemical Immunology and Allergy, vol. 94, pp. 189-200, 2008.

[28] B. N. Lambrecht and H. Hammad, "Biology of lung dendritic cells at the origin of asthma," Immunity, vol. 31, no. 3, pp. 412-424, 2009.

[29] C. J. Suarez, N. J. Parker, and P. W. Finn, "Innate immune mechanism in allergic asthma," Current Allergy and Asthma Reports, vol. 8, no. 5, pp. 451-459, 2008.

[30] P. G. Holt, "The role of airway dendritic cell populations in regulation of $\mathrm{T}$-cell responses to inhaled antigens: atopic asthma as a paradigm," Journal of Aerosol Medicine, vol. 15, no. 2, pp. 161-168, 2002.

[31] M. A. Gill, "The role of dendritic cells in asthma," Journal of Allergy and Clinical Immunology, vol. 129, no. 4, pp. 889-901, 2012.

[32] R. R. Dietert and J. T. Zelikoff, "Early-life environment, developmental immunotoxicology, and the risk of pediatric allergic disease including asthma," Birth Defects Research Part B, vol. 83, no. 6, pp. 547-560, 2008.

[33] P. G. Holt and P. D. Sly, "Prevention of adult asthma by early intervention during childhood: potential value of new generation immunomodulatory drugs," Thorax, vol. 55, no. 8, pp. 700-703, 2000.

[34] S. Awasthi, R. Wolf, and G. White, "Ontogeny and phagocytic function of baboon lung dendritic cells," Immunology and Cell Biology, vol. 87, no. 5, pp. 419-427, 2009.

[35] S. Awasthi and J. Cropper, "Immunophenotype and functions of fetal baboon bone-marrow derived dendritic cells," Cellular Immunology, vol. 240, no. 1, pp. 31-40, 2006.

[36] S. Awasthi, R. Madhusoodhanan, and R. Wolf, "Surfactant protein-A and toll-like receptor-4 modulate immune functions of preterm baboon lung dendritic cell precursor cells," Cellular Immunology, vol. 268, no. 2, pp. 87-96, 2011.

[37] T. Tschernig, V. C. De Vries, A. S. Debertin et al., "Density of dendritic cells in the human tracheal mucosa is age dependent and site specific," Thorax, vol. 61, no. 11, pp. 986991, 2006.

[38] T. Tschernig, A. S. Debertin, F. Paulsen, W. J. Kleemann, and R. Pabst, "Dendritic cells in the mucosa of the human trachea are not regularly found in the first year of life," Thorax, vol. 56, no. 6, pp. 427-431, 2001.

[39] R. R. Dietert, "Developmental immunotoxicity (DIT) in drug safety testing: matching DIT testing to adverse outcomes and childhood disease risk," Current Drug Safety, vol. 3, no. 3, pp. 216-226, 2008.

[40] R. R. Dietert and M. S. Piepenbrink, "The managed immune system: protecting the womb to delay the tomb," Human and Experimental Toxicology, vol. 27, no. 2, pp. 129-134, 2008.

[41] D. Gao, T. K. Mondal, and D. A. Lawrence, "Lead effects on development and function of bone marrow-derived dendritic cells promote Th2 immune responses," Toxicology and Applied Pharmacology, vol. 222, no. 1, pp. 69-79, 2007.

[42] M. Porter, M. Karp, S. Killedar et al., "Diesel-enriched particulate matter functionally activates human dendritic cells," American Journal of Respiratory Cell and Molecular Biology, vol. 37, no. 6, pp. 706-719, 2007.

[43] D. Gentile, J. Howe-Adams, J. Trecki, A. Patel, B. Angelini, and D. Skoner, "Association between environmental tobacco smoke and diminished dendritic cell interleukin 10 production during infancy," Annals of Allergy, Asthma and Immunology, vol. 92, no. 4, pp. 433-437, 2004.

[44] M. A. Williams, C. Cheadle, T. Watkins et al., "TLR2 and TLR4 as potential biomarkers of environmental particulate matter exposed human myeloid dendritic cells," Biomark Insights, vol. 2, pp. 226-240, 2007.

[45] G. F. G. Bezemer, S. M. Bauer, G. Oberdörster et al., "Activation of pulmonary dendritic cells and Th2-type inflammatory responses on instillation of engineered, environmental diesel emission source or ambient air pollutant particles in vivo," Journal of Innate Immunity, vol. 3, no. 2, pp. 150-166, 2011.

[46] M. A. Williams, T. Rangasamy, S. M. Bauer et al., "Disruption of the transcription factor Nrf2 promotes pro-oxidative dendritic cells that stimulate Th2-like immunoresponsiveness 
upon activation by ambient particulate matter," Journal of Immunology, vol. 181, no. 7, pp. 4545-4559, 2008.

[47] H. Renz, P. Brandtzaeg, and M. Hornef, "The impact of perinatal immune development on mucosal homeostasis and chronic inflammation," Nature Reviews Immunology, vol. 12, no. 1, pp. 9-23, 2012.

[48] L. Jiao, X. Han, S. Wang et al., "Imprinted DC mediate the immune-educating effect of early-life microbial exposure," European Journal of Immunology, vol. 39, no. 2, pp. 469-480, 2009.

[49] M. R. Starkey, R. Y. Kim, E. L. Beckett et al., "Chlamydia muridarum lung infection in infants alters hematopoietic cells to promote allergic airway disease in mice," PLoS ONE, vol. 7, no. 8, Article ID e42588, 2012.

[50] E. L. Beckett, S. Phipps, M. R. Starkey et al., "TLR2, but not TLR4, is required for effective host defence against Chlamydia respiratory tract infection in early life," PLoS ONE, vol. 7, no. 6, Article ID e39460, 2012.

[51] X. Roux, A. Remot, A. Petit-Camurdan et al., "Neonatal lung immune responses show a shift of cytokines and transcription factors toward Th2 and a deficit in conventional and plasmacytoid dendritic cells," European Journal of Immunology, vol. 41, no. 10, pp. 2852-2861, 2011.

[52] A. Al-Garawi, R. Fattouh, F. Botelho et al., "Influenza A facilitates sensitization to house dust mite in infant mice leading to an asthma phenotype in adulthood," Mucosal Immunology, vol. 4, no. 6, pp. 682-694, 2011.

[53] H. Renz, "Asthma protection with bacteria-science or fiction?" Thorax, vol. 66, no. 9, pp. 744-745, 2011.

[54] P. G. Holt, D. H. Strickland, and P. D. Sly, "Virus infection and allergy in the development of asthma: what is the connection?" Current Opinion in Allergy and Clinical Immunology, vol. 12, no. 2, pp. 151-157, 2012.

[55] T. Brar, S. Nagaraj, and S. Mohapatra, "Microbes and asthma: the missing cellular and molecular links," Current Opinion in Pulmonary Medicine, vol. 18, no. 1, pp. 14-22, 2012.

[56] K. Bendelja, A. Gagro, A. Bace et al., "Predominant type2 response in infants with respiratory syncytial virus (RSV) infection demonstrated by cytokine flow cytometry," Clinical and Experimental Immunology, vol. 121, no. 2, pp. 332-338, 2000.

[57] S. M. Van Schaik, D. A. Tristram, I. S. Nagpal, K. M. Hintz, R. C. Welliver, and R. C. Welliver, "Increased production of IFN$\gamma$ and cysteinyl leukotrienes in virus- induced wheezing," Journal of Allergy and Clinical Immunology, vol. 103, no. 4, pp. 630-636, 1999.

[58] R. P. Garofalo, J. Patti, K. A. Hintz, V. Hill, P. L. Ogra, and R. C. Welliver, "Macrophage inflammatory protein-1 $\alpha$ (not T helper type 2 cytokines) is associated with severe forms of respiratory syncytial virus bronchiolitis," Journal of Infectious Diseases, vol. 184, no. 4, pp. 393-399, 2001.

[59] L. Bont, C. J. Heijnen, A. Kavelaars et al., "Local interferon$\gamma$ levels during respiratory syncytial virus lower respiratory tract infection are associated with disease severity," Journal of Infectious Diseases, vol. 184, no. 3, pp. 355-358, 2001.

[60] J. P. Legg, I. R. Hussain, J. A. Warner, S. L. Johnston, and J. O. Warner, "Type 1 and type 2 cytokine imbalance in acute respiratory syncytial virus bronchiolitis," American Journal of Respiratory and Critical Care Medicine, vol. 168, no. 6, pp. 633-639, 2003.

[61] M. A. Gill, A. K. Palucka, T. Barton et al., "Mobilization of plasmacytoid and myeloid dendritic cells to mucosal sites in children with respiratory syncytial virus and other viral respiratory infections," Journal of Infectious Diseases, vol. 191, no. 7, pp. 1105-1115, 2005.

[62] M. A. Gill, K. Long, T. Kwon et al., "Differential recruitment of dendritic cells and monocytes to respiratory mucosal sites in children with influenza virus or respiratory syncytial virus infection," Journal of Infectious Diseases, vol. 198, no. 11, pp. 1667-1676, 2008.

[63] P. M. A. De Graaff, E. C. De Jong, T. M. Van Capel et al., "Respiratory syncytial virus infection of monocyte-derived dendritic cells decreases their capacity to activate CD4 T cells," Journal of Immunology, vol. 175, no. 9, pp. 5904-5911, 2005.

[64] T. P. Welliver, R. P. Garofalo, Y. Hosakote et al., "Severe human lower respiratory tract illness caused by respiratory syncytial virus and influenza virus is characterized by the absence of pulmonary cytotoxic lymphocyte responses," Journal of Infectious Diseases, vol. 195, no. 8, pp. 1126-1136, 2007.

[65] H. Wang, N. Peters, and J. Schwarze, "Plasmacytoid dendritic cells limit viral replication, pulmonary inflammation, and airway hyperresponsiveness in respiratory syncytial virus infection," Journal of Immunology, vol. 177, no. 9, pp. 62636270, 2006

[66] J. J. Smit, D. M. Lindell, L. Boon, M. Kool, B. N. Lambrecht, and N. W. Lukacs, "The balance between plasmacytoid DC versus conventional DC determines pulmonary immunity to virus infections," PLoS ONE, vol. 3, no. 3, Article ID e1720, 2008.

[67] D. J. Nelson, C. McMenamin, A. S. McWilliam, M. Brenan, and P. G. Holt, "Development of the airway intraepithelial dendritic cell network in the rat from class II major histocompatibility (Ia)-negative precursors: differential regulation of Ia expression at different levels of the respiratory tract," The Journal of Experimental Medicine, vol. 179, no. 1, pp. 203-212, 1994.

[68] K. Hamada, C. A. Goldsmith, A. Goldman, and L. Kobzik, "Resistance of very young mice to inhaled allergen sensitization is overcome by coexposure to an air-pollutant aerosol," American Journal of Respiratory and Critical Care Medicine, vol. 161, no. 4, part 1, pp. 1285-1293, 2000.

[69] F. M. Hofman, J. A. Danilovs, and C. R. Taylor, "HLA-DR (Ia)-positive dendritic-like cells in human fetal nonlymphoid tissues," Transplantation, vol. 37, no. 6, pp. 590-594, 1984.

[70] K. M. McCarthy, J. L. Gong, J. R. Telford, and E. E. Schneeberger, "Ontogeny of Ia+ accessory cells in fetal and newborn rat lung," American Journal of Respiratory Cell and Molecular Biology, vol. 6, no. 3, pp. 349-356, 1992.

[71] L. S. Van Winkle, M. V. Fanucchi, L. A. Miller et al., "Epithelial cell distribution and abundance in rhesus monkey airways during postnatal lung growth and development," Journal of Applied Physiology, vol. 97, no. 6, pp. 2355-2363, 2004.

[72] C. H. GeurtsvanKessel and B. N. Lambrecht, "Division of labor between dendritic cell subsets of the lung," Mucosal Immunology, vol. 1, no. 6, pp. 442-450, 2008.

[73] K. Flurkey, J. M. Currer, and D. E. Harrison, "Mouse models in aging research," in The Mouse in Biomedical Research, J. G. Fox, M. T. Davisson, F. W. Quimby, S. W. Barthold, C. E. Newcomer, and A. L. Smith, Eds., p. 63772, American College Laboratory Animal Medicine, Elsevier, Burlington, Mass, USA, 2007.

[74] C. G. Plopper and D. M. Hyde, "The non-human primate as a model for studying COPD and asthma," Pulmonary 
Pharmacology and Therapeutics, vol. 21, no. 5, pp. 755-766, 2008.

[75] J. Gordon, G. Grafton, P. M. Wood, M. Larché, and R. J. Armitage, "Modelling the human immune response: can mice be trusted?" Current Opinion in Pharmacology, vol. 1, no. 4, pp. 431-435, 2001.

[76] J. Mestas and C. C. W. Hughes, "Of mice and not men: differences between mouse and human immunology," Journal of Immunology, vol. 172, no. 5, pp. 2731-2738, 2004.

[77] M. Rehli, "Of mice and men: species variations of Toll-like receptor expression," Trends in Immunology, vol. 23, no. 8, pp. 375-378, 2002.

[78] M. Collin, V. Bigley, M. Haniffa, and S. Hambleton, "Human dendritic cell deficiency: the missing ID?" Nature Reviews Immunology, vol. 11, no. 9, pp. 575-583, 2011.

[79] F. Geissmann, "The origin of dendritic cells," Nature Immunology, vol. 8, no. 6, pp. 558-560, 2007.

[80] K. Shortman and S. H. Naik, "Steady-state and inflammatory dendritic-cell development," Nature Reviews Immunology, vol. 7, no. 1, pp. 19-30, 2007.

[81] M. V. Avdalovic, L. F. Putney, E. S. Schelegle et al., "Vascular remodeling is airway generation-specific in a primate model of chronic asthma," American Journal of Respiratory and Critical Care Medicine, vol. 174, no. 10, pp. 1069-1076, 2006.

[82] E. S. Schelegle, L. J. Gershwin, L. A. Miller et al., "Allergic asthma induced in rhesus monkeys by house dust mite (Dermatophagoides farinae)," American Journal of Pathology, vol. 158, no. 1, pp. 333-341, 2001.

[83] N. E. Schlabritz-Loutsevitch, G. B. Hubbard, M. J. Dammann et al., "Normal concentrations of essential and toxic elements in pregnant baboons and fetuses (Papio species)," Journal of Medical Primatology, vol. 33, no. 3, pp. 152-162, 2004.

[84] B. F. Barrier, E. J. Dick, S. D. Butler, and G. B. Hubbard, "Endometriosis involving the ileocaecal junction with regional lymph node involvement in the baboon-striking pathological finding identical between the human and the baboon: a case report," Human Reproduction, vol. 22, no. 1, pp. 272-274, 2007.

[85] L. D. Giavedoni, N. Schlabritz-Loutsevitch, V. L. Hodara et al., "Phenotypic changes associated with advancing gestation in maternal and fetal baboon lymphocytes," Journal of Reproductive Immunology, vol. 64, no. 1-2, pp. 121-132, 2004.

[86] N. E. Schlabritz-Loutsevitch, G. B. Hubbard, P. A. Frost et al., "Abdominal pregnancy in a baboon: a first case report," Journal of Medical Primatology, vol. 33, no. 1, pp. 55-59, 2004.

[87] N. Goncharov, G. Katzija, and T. Todua, "Peripheral plasma levels of 12 steroids during pregnancy in the baboon (Papio hamadryas)," European Journal of Obstetrics Gynecology and Reproductive Biology, vol. 11, no. 3, pp. 201-208, 1980.

[88] N. Goncharov, T. Aso, and Z. Cekan, "Hormonal changes during the menstrual cycle of the baboon (Papio hamadryas)," Acta Endocrinologica, vol. 82, no. 2, pp. 396-412, 1976.

[89] L. R. Sibal and K. J. Samson, "Nonhuman primates: a critical role in current disease research," ILAR Journal, vol. 42, no. 2, pp. 74-84, 2001.

[90] J. J. Coalson, V. T. Winter, T. Siler-Khodr, and B. A. Yoder, "Neonatal chronic lung disease in extremely immature baboons," American Journal of Respiratory and Critical Care Medicine, vol. 160, no. 4, pp. 1333-1346, 1999.

[91] M. H. Shearer, R. D. Dark, J. Chodosh, and R. C. Kennedy, "Comparison and characterization of immunoglobulin G subclasses among primate species," Clinical and Diagnostic Laboratory Immunology, vol. 6, no. 6, pp. 953-958, 1999.
[92] J. W. Hampton and C. Matthews, "Similarities between baboon and human blood clotting," Journal of Applied Physiology, vol. 21, no. 6, pp. 1713-1716, 1966.

[93] I. Gomes, T. T. Sharma, N. Mahmud et al., "Highly abundant genes in the transcriptosome of human and baboon CD34 antigen-positive bone marrow cells," Blood, vol. 98, no. 1, pp. 93-99, 2001.

[94] A. Denjean, B. Arnoux, and R. Masse, "Acute effects of intratracheal administration of platelet-activating factor in baboons," Journal of Applied Physiology, vol. 55, no. 3, pp. 799-804, 1983.

[95] S. Awasthi, J. J. Coalson, E. Crouch, F. Yang, and R. J. King, "Surfactant proteins A and D in premature baboons with chronic lung injury (bronchopulmonary dysplasia): evidence for an inhibition of secretion," American Journal of Respiratory and Critical Care Medicine, vol. 160, no. 3, pp. 942-949, 1999.

[96] S. Awasthi, J. J. Coalson, B. A. Yoder, E. Crouch, and R. J. King, "Deficiencies in lung surfactant proteins A and D are associated with lung infection in very premature neonatal baboons," American Journal of Respiratory and Critical Care Medicine, vol. 163, no. 2, pp. 389-397, 2001.

[97] S. Awasthi, J. Cropper, and K. M. Brown, "Developmental expression of Toll-like receptors-2 and -4 in preterm baboon lung," Developmental and Comparative Immunology, vol. 32, no. 9, pp. 1088-1098, 2008.

[98] D. N. Kotton, "Next-generation regeneration: the hope and hype of lung stem cell research," American Journal of Respiratory and Critical Care Medicine, vol. 185, no. 12, pp. 1255-1260, 2012.

[99] K. Bratke, M. Lommatzsch, P. Julius et al., "Dendritic cell subsets in human bronchoalveolar lavage fluid after segmental allergen challenge," Thorax, vol. 62, no. 2, pp. 168-175, 2007.

[100] J. Kayserova, I. Zentsova-Jaresova, V. Budinsky et al., "Selective increase in blood dendritic cell antigen-3-positive dendritic cells in bronchoalveolar lavage fluid in allergic patients," Scandinavian Journal of Immunology, vol. 75, no. 3, pp. 305-313, 2012.

[101] I. M. J. Beeren, M. S. De Bruin-Weller, C. Ra, I. Kok, C. A. F. M. Bruinzeel-Koomen, and E. F. Knol, "Expression of FceRI on dendritic cell subsets in peripheral blood of patients with atopic dermatitis and allergic asthma," Journal of Allergy and Clinical Immunology, vol. 116, no. 1, pp. 228-229, 2005.

[102] J. M. Tunon-De-Lara, A. E. Redington, P. Bradding et al., "Dendritic cells in normal and asthmatic airways: expression of the a subunit of the high affinity immunoglobulin E receptor (FceRI- $\alpha)$," Clinical and Experimental Allergy, vol. 26, no. 6, pp. 648-655, 1996.

[103] M. Spears, C. Mcsharry, I. Donnelly et al., "Peripheral blood dendritic cell subtypes are significantly elevated in subjects with asthma," Clinical and Experimental Allergy, vol. 41, no. 5, pp. 665-672, 2011.

[104] B. Dua, R. M. Watson, G. M. Gauvreau, and P. M. O’Byrne, "Myeloid and plasmacytoid dendritic cells in induced sputum after allergen inhalation in subjects with asthma," Journal of Allergy and Clinical Immunology, vol. 126, no. 1, pp. 133-139, 2010.

[105] B. Foster, D. D. Metcalfe, and C. Prussin, "Human dendritic cell 1 and dendritic cell 2 subsets express FceRI: correlation with serum IgE and allergic asthma," Journal of Allergy and Clinical Immunology, vol. 112, no. 6, pp. 1132-1138, 2003.

[106] M. M. Hagendorens, D. G. Ebot, A. J. Schuerwegh et al., "Differences in circulating dendritic cell subtypes in cord 
blood and peripheral blood of healthy and allergic children," Clinical and Experimental Allergy, vol. 33, no. 5, pp. 633-639, 2003.

[107] G. M. Möller, S. E. Overbeek, C. G. Van Helden-Meeuwsen et al., "Increased numbers of dendritic cells in the bronchial mucosa of atopic asthmatic patients: downregulation by inhaled corticosteroids," Clinical and Experimental Allergy, vol. 26, no. 5, pp. 517-524, 1996.

[108] F. L. Jahnsen, F. Lund-Johansen, J. F. Dunne, L. Farkas, R. Haye, and P. Brandtzaeg, "Experimentally induced recruitment of plasmacytoid (CD123high) dendritic cells in human nasal allergy," Journal of Immunology, vol. 165, no. 7, pp. 4062-4068, 2000.

[109] H. Matsuda, T. Suda, H. Hashizume et al., "Alteration of balance between myeloid dendritic cells and plasmacytoid dendritic cells in peripheral blood of patients with asthma," American Journal of Respiratory and Critical Care Medicine, vol. 166, no. 8, pp. 1050-1054, 2002.

[110] J. W. Upham, G. Zhang, A. Rate et al., "Plasmacytoid dendritic cells during infancy are inversely associated with childhood respiratory tract infections and wheezing," Journal of Allergy and Clinical Immunology, vol. 124, no. 4, pp. 707713.e2, 2009.

[111] A. Faith, N. Singh, E. Chevretton et al., "Counter regulation of the high affinity IgE receptor, Fc $\eta$ RI, on human airway dendritic cells by IL-4 and IL-10," Allergy, vol. 64, no. 11, pp. 1602-1607, 2009. 


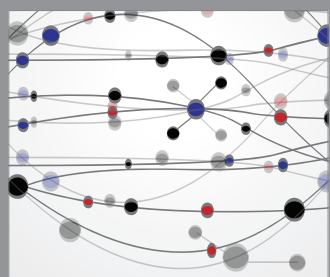

The Scientific World Journal
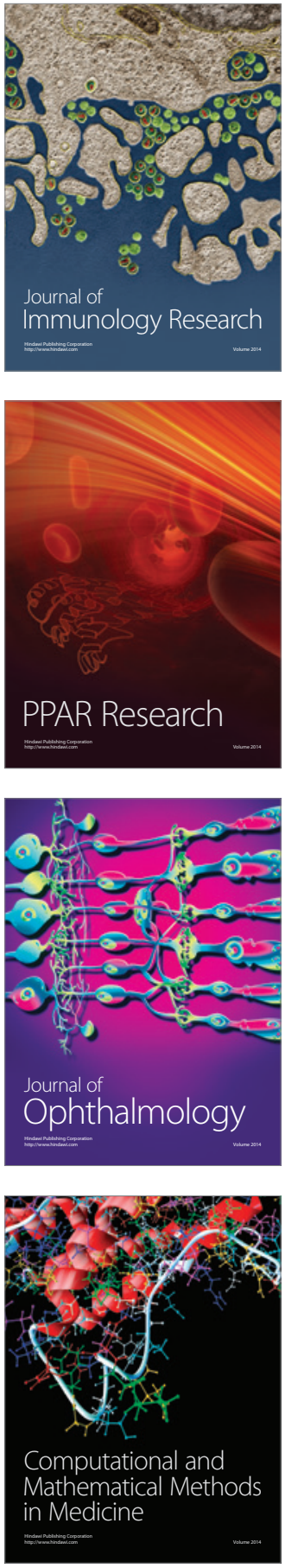

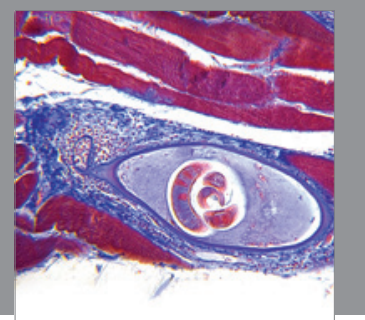

Gastroenterology

Research and Practice
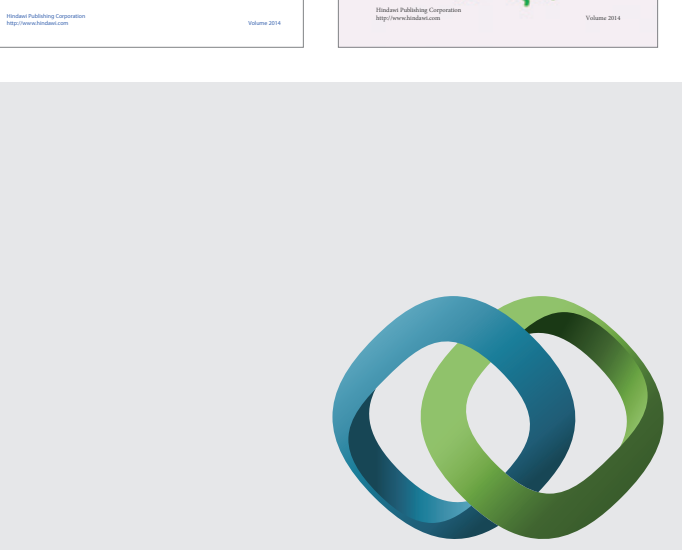

\section{Hindawi}

Submit your manuscripts at

http://www.hindawi.com
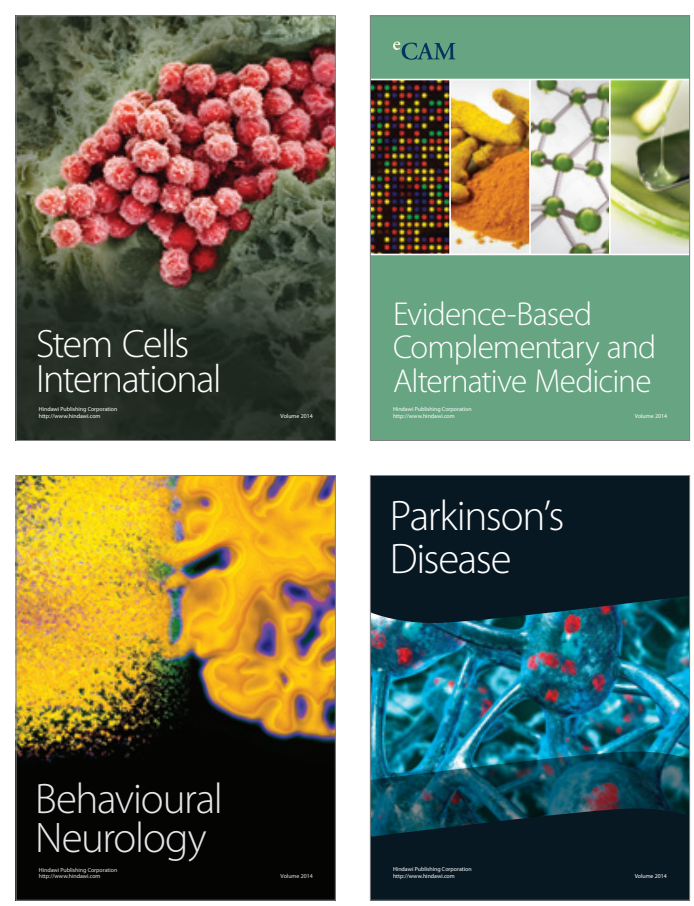

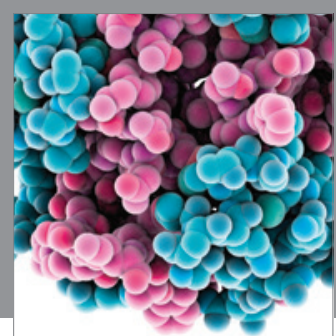

Journal of
Diabetes Research

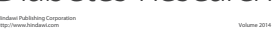

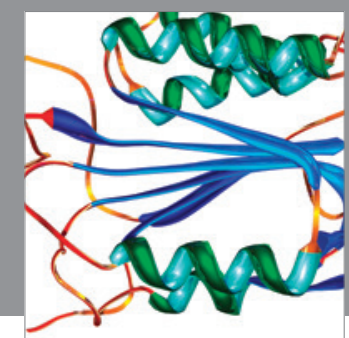

Disease Markers
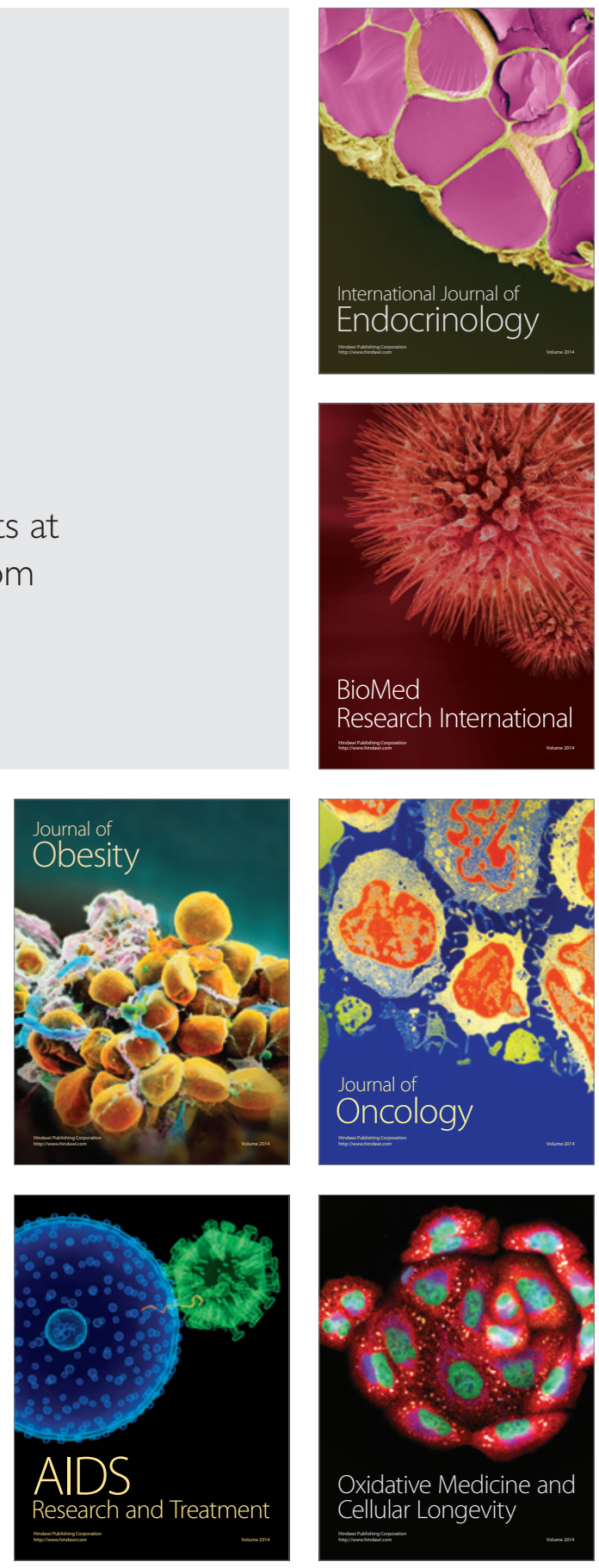